\title{
Covid-19 Infection in a Case of Transfusion Dependent Beta Thalassemia Major - A Case Report and Review of Literature
}

\author{
Anamika Giri ${ }^{1}$, Sameera Dronamraju ${ }^{2}$, Sourya Acharya ${ }^{3}$, Samarth Shukla ${ }^{4}$, Sunil Kumar ${ }^{5}$ \\ 1, 2,3,5 Department of Medicine, Datta Meghe Institute of Medical Sciences, Sawangi Meghe, Wardha, Maharashtra, India, \\ ${ }^{4}$ Department of Pathology, Datta Meghe Institute of Medical Sciences, Sawangi Meghe, Wardha, Maharashtra, India.
}

\section{INTRODUCTION}

Thalassemia, derived from Thalassa [Greek: Sea] refers to a cluster of hereditary haemoglobinopathies initially reported in areas of Sub-Saharan Africa, the Indian subcontinent, Southeast Asia, and the Mediterranean where malaria was (or is) endemic. Currently, it is the most frequently recognized haemoglobinopathy, and has been correlated with a plethora of immune system changes such as neutropenia, natural killer cell dysfunctions, increased activity of CD8 suppressor cells, along with disturbances in macrophage function, chemotaxis, phagocytosis, and interferons INF$\gamma$ production. ${ }^{1}$

The reduction in the alpha or beta chain that leads to the production of haemoglobin is the key pathophysiology behind thalassemia. Therefore, weakened red blood cells can contribute to inadequate erythropoiesis and haemolytic anaemia. Thalassemia diseases are known as transfusion-dependent or non-dependent according to either their beta-globin chain genotype or their clinical path. The most serious type of thalassemia is beta-thalassemia major, and it is transfusiondependent, presenting in early infancy. ${ }^{2}$

A diagnosis of $\beta$-thalassemia major (BTM) means lifelong, regular transfusions, supplemented with adequate iron chelation therapy for the patient. ${ }^{3}$ This also means that unless adequate blood screening and hygiene procedures are in place, patients with BTM are at a risk of transfusion transmitted infections (TTI). ${ }^{4}$

Many countries have been prompted by the spread of Covid-19 infection to shutter routine outpatient coronary health care units before the pandemic is over, which also extends to people with thalassemia, who require regular follow-ups. While Covid-19 infection rates remain high, patients with thalassemia with planned cardiovascular examinations are advised to delay their medical appointments for a minimum of 3 months. ${ }^{1}$ In addition, according to the statement published by the Thalassemia International Foundation on the $13^{\text {th }}$ of July, 2020, patients with hemoglobinopathies are at a greater risk in acquiring the Covid-19 infection, posing an additional challenge to the patients, families, and medical staff managing these disorders. ${ }^{5}$ Thalassaemic patients have reduced levels of protein $\mathrm{S}$ and protein $\mathrm{C}$; elevated aggregation of platelets; and recruitment of monocytes, granulocytes, and endothelial cells. Patients of thalassemia also show elevated markers of platelet and coagulation activation, even in the absence of major thromboembolic events. An increase in D-Dimer levels is also seen in most Covid-19 patients who are hospitalised, attributable to the underlying inflammatory process. ${ }^{1}$

A variety of questions have been raised during the ongoing Covid-19 pandemic with respect to diagnostic and clinical approaches towards this particular population. The precise path of infection with Covid-19 in those patients has yet to be elucidated. ${ }^{2}$ The progression of Covid-19 infection in patients with hemoglobinopathy in general and thalassemia in particular is not fully known and is still an area of discussion and under review. We report a case of a 30-year-old male patient, a known case of beta thalassemia major on maintenance transfusion and iron chelation therapy who acquired Covid-19 infection.

\begin{abstract}
Corresponding Author:
Dr. Sourya Acharya,

Professor and Head of Department, Department of Medicine, Datta Meghe Institute of Medical Sciences, Sawangi Meghe, Wardha, Maharashtra, India. E-mail: souryaacharya74@gmail.com
\end{abstract}

DOI: $10.14260 /$ jemds/2021/288

How to Cite This Article:

Giri A, Dronamraju $S$, Acharya $S$, et al. Covid-19 infection in a case of transfusion dependent beta thalassemia major - a case report and review of literature. J Evolution Med Dent Sci 2021;10(18):1365-1368, DOI: 10.14260/jemds/2021/288

Submission 14-12-2020,

Peer Review 28-02-2021,

Acceptance 06-03-2021,

Published 03-05-2021.

Copyright (C) 2021 Anamika Giri et al. This is an open access article distributed under Creative Commons Attribution License [Attribution 4.0 International (CC BY 4.0)] 


\section{PRESENTATION OF CASE}

A 30-year-old male, a known case of transfusion-dependent beta-thalassemia major was presented to us with chief complaints of fever for 4 days, cough since 3 days along with headache and myalgia. Patient was apparently alright 4 days ago when he started experiencing fever which was moderate grade and continuous in nature but not associated with chills and rigours. There was no diurnal variation. Fever was not relieved on taking antipyretics.

Patient also complained of cough since 3 days which was dry, without any expectoration, no diurnal or positional variation without any wheezing. He had headache and myalgia. There was no history of breathlessness, expectoration, haemoptysis, anosmia, chest pain, abdominal pain, diarrhoea. Patient was a known case of beta thalassemia major since 15 years and was on regular transfusion therapy twice a month along with iron chelation therapy. In view of the presenting complaints urgent RT-PCR for Covid-19 was done which came to be positive, all routine investigations were done, and patient was admitted in Covid positive mobile intensive care unit (MICU) for further management.

On examination, patient was conscious and well oriented to time, place and person. Patient was malnourished. Temperature was $100^{\circ} \mathrm{F}$, pulse 110 per min while systolic / diastolic pressure was 110 / $70 \mathrm{~mm}$ of $\mathrm{Hg}$ in right arm supine position. Respiratory Rate was 20 per minute (abdominothoracic). Patient's saturation was $93 \%$ on breathing ambient room air. Patient's saturation improved to $98 \%$ after putting him on oxygen support (2L / Min). Pallor present, icterus present. There were no signs of lymphadenopathy, cyanosis or clubbing. Jugular venous pressure (JVP) was normal.

On systemic examination, respiratory system examination was normal, cardiovascular system as well as per abdomen system examination was normal.

Patient's blood investigations comprised of not just routine investigations but also investigations falling under the Covid panel including serum lactate dehydrogenase (LDH), serum VIT D, serum $C$ reactive protein (CRP), erythrocyte sedimentation rate (ESR), serum uric acid and others.

Complete blood count with peripheral smear showed Haemoglobin (Hb)-8.6 g / dL, mean corpuscular volume (MCV)-91fL, total leukocyte count (TLC)-19,100 / microlitre, Platelets-2,50,000 / microlitre.

Patient's coagulation profile showed prothrombin time of 14.90 seconds and activated partial thromboplastin (APTT) of 32.60 seconds. E.S.R was $35 \mathrm{~mm} /$ hour in the first hour. Peripheral smear showed predominantly normocytic mildly hypochromic RBCs, adequate platelets on smear, and no hemoparasite. Mild anisopoikilocytosis (pencil cells) and target cells were seen in the RBCs. Arterial blood gas analysis showed hypoxia with $\mathrm{PaO} 2$ of $80 \mathrm{mmHg}$ and a metabolic acidotic picture. Serum calcium levels were $9.5 \mathrm{mg} / \mathrm{dl}$. The CRP (quantitative) was $2.9 \mathrm{mg} / \mathrm{L}$ (normal: less than $10 \mathrm{mg} /$ litre). D-dimer concentration was raised to; $-1.5 \mathrm{mcg} / \mathrm{mL}$ (the reference concentration of D-dimer is $<0.4 \mathrm{mcg} / \mathrm{mL}$ ). Serum ferritin levels were found to be $13000 \mathrm{ng} / \mathrm{Dl}$ (normal 20 - 250. $\mathrm{Ng} / \mathrm{dL}$ ). KFT-urea - $45 \mathrm{mg} / \mathrm{dL}$, creatinine - $0.9 \mathrm{mg} / \mathrm{dL}$. Sodium levels were slightly reduced to $131 \mathrm{mEq} /$ L. Potassium levels were slightly raised (5.9 mmol / L). Serum LDH - 277 U / L (normal range: 140 - 280 units / litre).

Liver Function Test revealed Albumin (3.5 g / dL), conjugated \& unconjugated bilirubin (1.1 and $0.7 \mathrm{mg} / \mathrm{dL}$ respectively), globulin (3.6 g / dL), SGPT (23 U / L), SGOT (36 $\mathrm{U} / \mathrm{L}$ ), total protein $(7.1 \mathrm{~g} / \mathrm{dL})$, and total bilirubin. (1.8 $\mathrm{mg} /$ dL)

Uric Acid levels were found to be $2.3 \mathrm{mg} / \mathrm{dl}$ (normal range: $2.5-7 \mathrm{mg} / \mathrm{dl}$ ) and Vitamin D levels were $21.2 \mathrm{ng} / \mathrm{ml}$ (normal range: $20-50 \mathrm{ng} / \mathrm{ml}$ ). CXR showed cardiomegaly (Figure 1)

High resolution computed tomography (HRCT) scan of Thorax was done, and it was suggestive of HRCT severity score of 0 / 25 indicating no obvious abnormality in bilateral lung fields. (Figure 2)
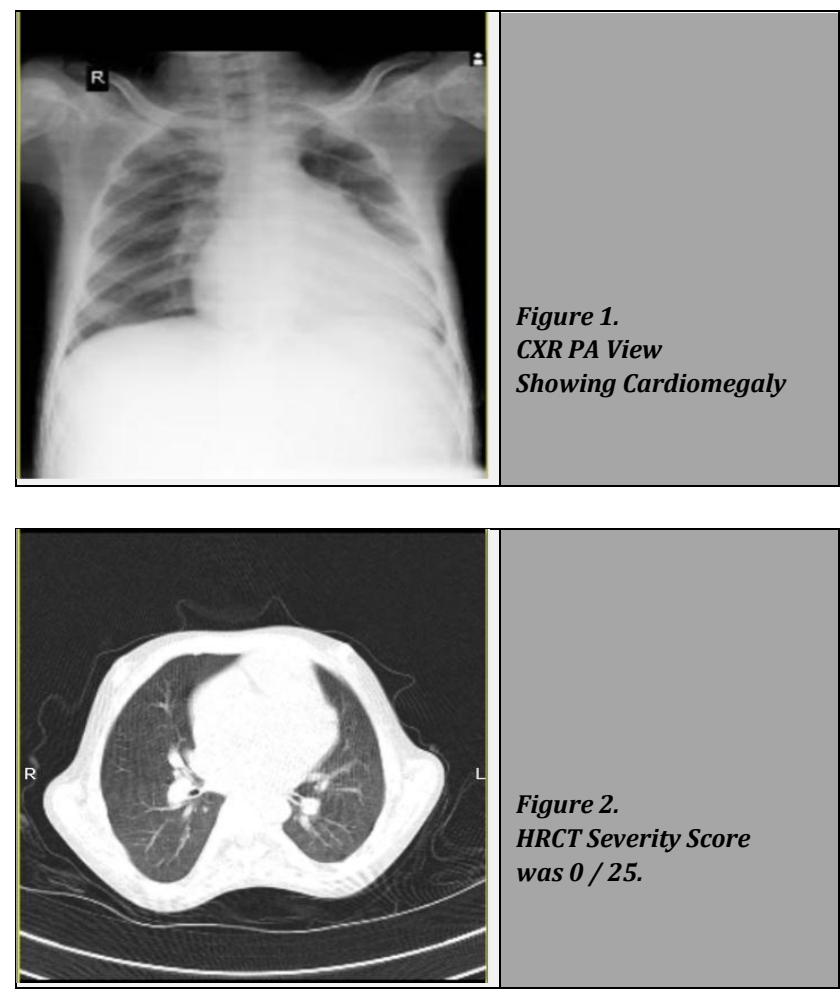

\section{DISCUSSION OF MANAGEMENT}

Patient was started on all supportive management including oxygen support (2 litres / minute) via a face mask. Patient received a full course of antiviral injectable Remdesivir $200 \mathrm{mg}$ in $100 \mathrm{ml}$ NS IV stat followed by $100 \mathrm{mg}$ in $100 \mathrm{ml} \mathrm{NS}$ IV OD for 4 days. Inj. doxycycline $100 \mathrm{mg}$ iv $\mathrm{BD}$ for 5 days, Inj dexamethasone $6 \mathrm{mg}$ IV OD for 5 Days. Since patient's D-Dimer was raised patient was started on Inj. Enoxaparin $0.4 \mathrm{ml} \mathrm{S} \mathrm{/} \mathrm{C}$ bid for 5 days. Meanwhile patient underwent his regular Thalassemia therapy and was transfused with 2 units of blood.

During the course of his stay in the hospital patient was closely monitored for any signs of deterioration. Patient was maintaining satisfactory saturation on room air on this treatment and there were no active complaints.

After 14 days, a repeat swab for Covid-19 (RT-PCR) was sent which came negative and as patient had no active complaints. His repeat D-dimer report showed a value of 0.4 $\mathrm{mcg} / \mathrm{ml}$. Repeat serum Ferritin level was $300 \mathrm{ng} / \mathrm{mL}$. Patient 
was discharged on Tab Desirox OD, Tab Limcee, Tab Zinc, Tab Folic Acid, Tab Pantoprazole OD and was advised blood transfusion every 15 days. Patient was also advised isolation and home quarantine for 14 days.

\section{DISCUSSION}

Thalassemia beta is a genetic disorder caused due to defective haemoglobin synthesis, leading to chronic haemolysis and premature death of bone marrow erythrocytes. This leads to chronic, severe anaemia and bone marrow expansion leading to serious complications if left unchecked. ${ }^{2}$ Thalassemia diseases are categorized into beta-thalassemia major (BTM), beta-thalassemia intermediate (BTI), or beta-thalassemia minor based on clinical and genetic history (carriers). Thalassemia has been divided into two major groups in practise, namely transfusion-dependent-thalassemia (TDT) or non-transfusion-dependent-thalassemia (NTDT).

While blood transfusion is considered the corner stone in the BTM management strategy, due to major complications such as iron overload, transfusion-transmitted infections (TTIs), and antibody production, it is still a troublesome concern. Therefore, in increasing life expectancy and reducing morbidities due to iron overload, iron chelation therapy (ICT) is of essential importance. After 15 days of hospital stay the laboratory investigations of the patient were within normal ranges.

Several factors increase the risk of infections, particularly immune system dysfunction, iron overload, and multiple blood transfusions, in thalassemia disorders. In addition, splenectomy and adrenal hypofunction, especially in older patients, may play a role in increased infections. ${ }^{6}$ In BTM patients, the precise clinical path of Covid-19 infection is not yet well recognised.

Thrombosis and an increased need for anticoagulation have been correlated with Covid-19. Patients of hemoglobinopathy, though, are at a greater risk of bleeding, so they should be closely watched for bleeding. Physicians should weigh the costs and benefits with a patient in order to determine whether anticoagulation is indicated or not. Anticoagulation was granted to our patient, as he had symptomatic Covid-19 infection path, no chance of thrombosis as per experience, review, and primary investigations.

There is no evidence available on cases of Covid-19 in patients with thalassemia. Motta I et al. ${ }^{7}$ recorded the experience of 11 cases of thalassemia patients from the Italian Network of Centers for Hemoglobinopathies and concluded that, while preliminary findings did not suggest an increased severity of Covid-19 in patients with thalassemia, they did not indicate an increased severity of Covid $-19^{8}$. In the other side, we identify the case of a 30-year-old man with Covid-19 pneumonia thalassemia, treated with a mixture of antibiotics and oxygen therapy. The patient had an increase in transfusion demand during hospitalisation, as it usually happens in patients with TDT during the infectious phase, with a reduction of approximately two grams of haemoglobin detected. We indicate that thalassemia is not inherently a source of aggravation of the clinical course in Covid-19; however, some main considerations, such as the status of anaemia, the probable pathogenic effect of the virus on haemoglobin, and the hypercoagulable state, must be addressed when treating these cases in order to avoid any complications in patients with TDT. Chen $\mathrm{N}$ et al. ${ }^{9}$ reported a drop in the haemoglobin levels of most patients infected with Covid-19, and an improvement in the serum ferritin and haem index values.

\section{CONCLUSIONS}

One of the most common hemoglobinopathies is betathalassemia major. There is a wide variety of presentations and complications for people with this condition, often from iron overload. A significant means of minimizing problems is ICT. One of the most worthwhile questions to ask is whether or not these patients can be deemed to be at high risk of infection with Covid-19 and its complications during this pandemic. We need to make a definitive decision. We assume that screening for Covid-19 infection in patients with thalassemia is important as they may be asymptomatic.

In a male with BTM, we reported a smooth clinical path for infection with Covid-19. In order to know the precise course of Covid-19 in Thalassemia cases, additional data needs to be gathered, of course, even whether iron chelation therapy compliance has any part to play in minimizing the presenting symptoms. In addition, it is important to consider the particular side effects of chelator products, such as agranulocytosis, elevated risk of infection and acute kidney injury. Moreover, thalassemia is an additional risk of heart problems in patients with cardiac iron overload during serious infections. Due to the highest risk of extreme anaemia and thromboembolic complications, sufficient transfusion treatment, chelator discontinuation and the initiation of early anticoagulant therapy are important in patients with thalassemia.

Financial or other competing interests: None.

Disclosure forms provided by the authors are available with the full text of this article at jemds.com.

\section{REFERENCES}

[1] Aggeli C, Delicou S, Patsourakos D, et al. Concerns regarding the management of $\mathrm{B}$-thalassaemia patients in the era of COVID-19. EMJ Hematol 2020;8(1):100-4.

[2] Okar L, Ali M, Parengal J, et al. COVID-19 and thalassemia beta major in splenectomized patient: clinical case progression and literature review. Clin Case Rep 2020;8(12):2918-22.

[3] Canatan D, De Sanctis V. The medical concerns of patients with thalassemias at the time of COVID-19 outbreak: the personal experience and the international recommendations. Acta Biomed 2020;91(2):218-21.

[4] Shah FT, Sayani F, Trompeter S, et al. Challenges of blood transfusions in $\beta$-thalassemia. Blood Rev 2019;37:100588.

[5] Okar L, Aldeeb M, Ahmad R, et al. COVID-19 infection in thalassemia beta major: case series. International Journal of Case Reports 2020;4:161.

[6] Modell B, Khan M, Darlison M, et al. Improved survival of thalassaemia major in the $\mathrm{UK}$ and relation to $\mathrm{T} 2 *$ 
cardiovascular magnetic resonance. J Cardiovasc Magn Reson 2008;10(1):42.

[7] Motta I, De Amicis MM, Pinto VM, et al. SARS-CoV-2 infection in beta thalassemia: preliminary data from the Italian experience. Am J Hematol 2020;95(8):E198-9.

[8] Marziali M, Ribersani M, Losardo AA, et al. COVID-19 pneumonia and pulmonary microembolism in a patient with B-thalassemia major. Clin Case Rep 2020:8(12):3139-42.

[9] Chen N, Zhou M, Dong X, et al. Epidemiological and clinical characteristics of 99 cases of 2019 novel coronavirus pneumonia in Wuhan, China: a descriptive study. Lancet 2020;395(10223):507-13. 